University of Nebraska - Lincoln

DigitalCommons@University of Nebraska - Lincoln

Computer Programs for Temperature in Fins and Slab Bodies with the Method of Green's Functions

Kevin D. Cole

University of Nebraska-Lincoln, kcole1@unl.edu

Follow this and additional works at: https://digitalcommons.unl.edu/mechengfacpub

Part of the Mechanical Engineering Commons

Cole, Kevin D., "Computer Programs for Temperature in Fins and Slab Bodies with the Method of Green's Functions" (2004). Mechanical \& Materials Engineering Faculty Publications. 38.

https://digitalcommons.unl.edu/mechengfacpub/38

This Article is brought to you for free and open access by the Mechanical \& Materials Engineering, Department of at DigitalCommons@University of Nebraska - Lincoln. It has been accepted for inclusion in Mechanical \& Materials Engineering Faculty Publications by an authorized administrator of DigitalCommons@University of Nebraska Lincoln. 


\title{
Computer Programs for \\ Temperature in Fins and \\ Slab Bodies with the \\ Method of Green's
}

\section{Functions}

\author{
Kevin D. Cole
}

Mechanical Engineering Department, N104 Walter Scott Engineering Center, University of Nebraska-Lincoln, Lincoln, Nebraska 68508-0656; email kcole1@unl.edu

\begin{abstract}
Heat conduction in fins and slab bodies, a traditional undergraduate topic, is treated with the Green's function (GF) method. A variety of boundary conditions and heating conditions are included. Computer programs are described which are designed to improve student learning of the GF method. The programs are available from the author.
\end{abstract}

Keywords: heat conduction, convection heat transfer, Green's functions, open-source software, analytical solution

\section{Introduction}

This study is focused on heat transfer in fins and slab bodies by the method of Green's functions (GF). The method is powerful because a small set of GF can be used to find temperatures caused by many different boundary conditions and internal heating conditions. The method is systematic because if the GF is known, an expression for the temperature can be written immediately according to a straightforward algorithm. Unfortunately, the power of the GF method has proved to be something of a barrier to its widespread use. Discus- sions of the GF method generally involve a high level of abstraction, covering several differential equations, multiple coordinate systems, three-dimensional geometries, and so on. In this study, the discussion is limited to fins and slab bodies to make the GF method more accessible to undergraduate students.

Another barrier to wider use of the GF method has been the daunting task of finding the GF itself. This study emphasizes use of an existing collection of GF to solve engineering problems. This approach exposes students to a broader landscape of analytic solutions than is typically present in heat transfer textbooks, with a minimum time 
investment, at a mathematical level consistent with current undergraduate courses. The task of finding the GF for new applications may be deferred to an advanced course.

Next, a review of pertinent literature will be given in the areas of GF, fins, and computer programs for student learning in heat transfer. In the area of GF, the method was first applied to heat conduction early in the twentieth century by Carslaw [1]. Later Carslaw teamed with Jaeger to produce an enduring reference on analytical heat conduction and the GF method. The 1959 edition of their book has never gone out of print [2]. Perhaps subsequent researchers turned away from analytical methods as digital computers took center stage. However, in the last few years the GF method has been undergoing something of a revival, with several books entirely devoted to GF [3-6].

In the area of fins, the mathematical theory for heat conduction in fins was pioneered by Harper and Brown in 1922 [7] and refined by Garder in 1945 [8]. Jakob included a discussion of fins in his influential 1949 book [9], and since then fin analysis has been included in essentially every heat transfer text. There is an enormous literature of numerical solutions to heat transfer in fins which cannot be discussed here. However there is one paper that deserves mention for a discussion of fins with a variety of boundary conditions [10] solved by the GF method. The GF are computed numerically with a Galerkin-based integral method. In contrast, in the present paper the GF for fins are given as analytical expressions.

Next, the literature will be discussed in the area of computer programs designed for student learning in heat transfer. Some programs contain compilations of readyto-use formulas drawn chapter-by-chapter from a specific textbook (for example [11]). Other programs are intended to stand alone or to supplement a course on heat transfer. One such program for one-dimensional transient conduction in solids was created by Haji-Sheikh [12] for the DOS operating system. The program has a graphical user interface, and the numerical results are computed from Galerkin- based GF [13]. Another stand-alone program for one-dimensional conduction, written for the Windows operating system by Ribando and O'Leary [14], uses a finite-difference method for the numerical results.

Recently, programs to enhance student learning of heat transfer have begun appearing on the world wide web. A web-based program described by Somerton [15] contains Nusselt number correlations for predicting convection heat transfer. The correlations are classified by the type of convection present (forced, free, or mixed), the type of fluid flow present (external or internal), and the surface geometry. The correlations are drawn from established heat transfer textbooks, and full citations are given. Use of this system can enhance the ability of students to make good choices for predicting the behavior of physical systems.

The author maintains an internet site devoted to GF called the GF Library $[16,17]$. The purpose of the GF Library is to catalog known GF and to promote the GF method. The GF Library also serves as a supplement to a senior course in heat transfer and a graduate course in heat conduction. The GF are organized by equation type, coordinate system, body shape, and type of boundary conditions. Specific GF may be found through the table of contents or from a search of the site. The site also includes a brief introduction to the GF method, some examples, and supporting material.

This article makes two contributions. First, the method of GF is applied to heat conduction in fins and a comprehensive listing of the associated GF is given. Although fins were briefly discussed in our 1992 book on GF [5], to the author's knowledge there has been no treatment of fins that includes a complete listing of the GF for fins. Second, computer programs are described that provide expressions for analytical solutions for heat conduction in fins and slab bodies, topics traditionally included in an undergraduate course in heat transfer. Since the solutions are based on the GF method, a wide variety of heating conditions are treated. These programs are intended to improve student learning.

The remainder of the study is divided into sections, including: heat conduction in fins; the GF method; new computer programs for heat conduction; open-source software; computer display of math content; and, the conclusions.

\section{Heat Conduction in Fins}

Consider the steady temperature in a fin of uniform cross section, which satisfies the following second order, linear, differential equation:

$$
\frac{\mathrm{d}^{2} T}{\mathrm{~d} x^{2}}-m^{2}\left(T-T_{\infty}\right)+\frac{1}{k} g(x)=0 ; \quad x \text { in domain } R
$$

Here $m^{2}=h P /(k A)$ describes heat loss by convection from the side of the fin where $h$ is the heat transfer coefficient $\left(\mathrm{W} / \mathrm{m}^{2} / \mathrm{K}\right), P$ is the fin perimeter $(\mathrm{m}), A$ is the cross section area of the fin $\left(\mathrm{m}^{2}\right)$, and $k$ is the thermal conductivity $\left(\mathrm{W} / \mathrm{m}^{2} / \mathrm{K}\right)$ Term $g(x)$ represents volume energy generation within the fin (such as by electric heating or chemical reaction); this term is included to demonstrate the versatility of the GF method. Domain R may include $(\infty, \infty)$ for the infinite fin, $(0, \infty)$ for the semi-in- 
finite fin, or $(0, L)$ for the finite fin. The boundary conditions at the ends of the fin may be of several types, all of which are described by the following condition:

$$
\left.k_{i} \frac{\mathrm{d} T}{\mathrm{~d} n_{i}}\right|_{x_{i}}+h_{i}\left(T\left(x_{i}\right)-T_{\infty}\right)=f_{i} ; \quad i=1,2
$$

Here $x_{i}$ is located on the $i$ th boundary and direction $n_{i}$ is the outward normal on the $i$ th boundary. The general boundary condition (Equation 2) represents one of several boundary types, as follows: for type 1 , set $k_{i}=0$ and $h_{i}=1$ to give $T\left(x_{i}\right)=f_{i}$ (specified temperature); for type 2 , set $k_{i}=k$ and $h_{i}=0$ to give $k \mathrm{~d} T / \mathrm{d} n_{i}=f_{i}$ (specified heat flux); and, for type 3 , set $k_{i}=k$ and $h_{i}=h$ for specified convection. The boundary condition of type 0 describes the condition where there is no physical boundary, for example at $x \rightarrow \infty$ in a semi-infinite fin, where both $\mathrm{d} T /$ $\mathrm{d} x \rightarrow 0$ and $\left.T-T_{\infty}\right) \rightarrow 0$.

\section{Green's Function Method}

In this section, the temperature in the fin will be given with the GF method. Like many analytical solution techniques, the GF method requires that the differential equation be linear, the boundary conditions be linear, and that the boundary conditions be applied at regular boundaries, all of which apply to the fin problem given above. The GF itself represents the response of the fin to a point source of heat which may, with superposition, be used to construct the desired temperature solution. Other names for the GF include impulse response, fundamental solution, and influence function, among others.

The Dirac delta function, sometimes called the unit impulse function, is central to the GF method. The properties of the Dirac delta function that are needed for the GF method are given in Table 1.

The GF for the fin satisfies the following equations:

$$
\begin{gathered}
\frac{\mathrm{d}^{2} G}{\mathrm{~d} x^{2}}-m^{2} G=-\delta\left(x-x^{\prime}\right) ; \quad x \text { in domain } R \\
\left.k_{i} \frac{\mathrm{d} G}{\mathrm{~d} n_{i}}\right|_{x_{i}}+h_{i} G \mid x_{i}=0 ; \quad i=1,2
\end{gathered}
$$

Table 1. Properties of the Dirac Delta Function, $\delta\left(x-x^{\prime}\right)$

1. $\int_{R} \delta\left(x-x^{\prime}\right) \mathrm{d} x^{\prime}=1$

2. $\delta\left(x-x^{\prime}\right)=\left\{\begin{array}{lr}\infty & \text { as } x \rightarrow x^{\prime} \\ 0 & \text { otherwise }\end{array}\right.$

3. $\int_{R} F\left(x^{\prime}\right) \delta\left(x-x^{\prime}\right) \mathrm{d} x^{\prime}=F(x)$, the sifting property
Note that the above equations are similar to the temperature problem, except the energy generation term is replaced by a Dirac delta function and the boundary conditions are homogeneous. Most importantly, the boundary conditions must be of the same type as the specific temperature problem of interest. The GF $G\left(x \mid x_{0}\right)$ represents the heat transfer response at observation point $x$ to an infinitesimal heat source located at point $x_{0}$. An important property of the GF is that the GF is symmetric with respect to position: $G\left(x \mid x_{0}\right)=G\left(x_{0} \mid x\right)$ : This is called the reciprocity relation.

The temperature solution is constructed from a suitable distribution of the GF within the body so as to reproduce the heating conditions. The temperature in the fin is given by:

$$
T(x)-T_{\infty}=\frac{1}{k} \int g\left(x^{\prime}\right) G\left(x \mid x^{\prime}\right) \mathrm{d} x^{\prime}
$$

(for energy generation)

$$
+\sum_{i=1}^{2} \frac{f_{i}}{k_{i}} G\left(x \mid x_{i}^{\prime}\right)
$$

(for boundary of type 2 or 3 )

$$
-\left.\sum_{i=1}^{2} f_{j} \frac{\mathrm{d} G}{\mathrm{~d} n_{j}^{\prime}}\right|_{x^{\prime}=x_{j}}
$$

(for boundary of type 1 only)

This is the GF solution equation for steady heat conduction in the fin, for a derivation refer to the Appendix. The summation over $i$ is used to represent the contribution from boundaries of type 2 or 3 , and the summation over $j$ is used to represent the contribution from boundaries of type 1 . There are, of course, a total of two boundaries for fins of finite extent $(0<x<L)$. The above notation also applies to semi-infinite or infinite bodies, if one or zero boundary terms are included, respectively. The above expression represents the solution to a wide variety of fin problems, with different heating and cooling conditions, including internal heat generation. In the next section, a number system is introduced that is useful for keeping track of the many different possible fin solutions that can be treated by the GF method.

\section{Number System}

A number system is essential to identify various GF for storage and retrieval on a computer. The GF number has the form "X- -" where the $\mathrm{X}$ represents the coordinate system and the "- - " represent two digits, one each for the type of boundary conditions. For example, designation X12 represents a GF with a type 1 boundary at $x=0$ 
and a type 2 boundary at $x=L$. As another example, designation X10 represents a GF with a type 1 boundary at $x=0$ and the other boundary is nonphysical, that is, the fin is semi-infinite.

The number system also extends to precise descriptions of temperature distributions with the addition of descriptors for boundaries, generation, and initial conditions. Boundary information is identified by prefix "B," generation by prefix " $\mathrm{G}$," and initial condition by prefix "T." Numbers following the letter prefixes indicate the value specified. Number "0" denotes zero effect; number " 1 " denotes a constant effect; and a dash "-" denotes a nonconstant effect. For example, descriptor "B11" denotes a constant heating condition at each of two boundaries; and, "G-" denotes a nonconstant energy-generation effect in the body. An example is given later of the number system applied to a fin.

The number system also applies to transient heat conduction, to other coordinate systems, and to multidimensional problems. Further details of the number system may be found elsewhere [5].

\section{GF for Fins}

The GF needed for all the fin problems discussed above are designated XIJ where I, $\mathrm{J}=0,1,2,3$. The GF for these geometries is given by

$$
\begin{aligned}
G\left(x, x^{\prime}\right)= & \frac{S_{2}^{-}\left(S_{1}^{-} e^{-m\left(2 L-\left|x-x^{\prime}\right|\right)}+S_{1}^{+} e^{-m\left(2 L-x-x^{\prime}\right)}\right)}{2 m\left(S_{1}^{+} S_{2}^{+}-S_{1}^{-} S_{2}^{-} e^{-2 m L}\right)} \\
& +\frac{S_{2}^{+}\left(S_{1}^{+} e^{-m\left(\left|x-x^{\prime}\right|\right)}+S_{1}^{-} e^{-m\left(x+x^{\prime}\right)}\right)}{2 m\left(S_{1}^{+} S_{2}^{+}-S_{1}^{-} S_{2}^{-} e^{-2 m L}\right)}
\end{aligned}
$$

where the coefficients depend on the type of boundary conditions at boundaries $i=1,2$ and are given by:

$$
\begin{aligned}
& S_{i}^{+}= \begin{cases}1 & \text { side } i \text { is type } 0,1, \text { or } 2 \\
m L+B_{i} & \text { side } i \text { is type } 3\end{cases} \\
& S_{i}^{-}= \begin{cases}0 & \text { side } i \text { is type } 0 \\
-1 & \text { side } i \text { is type } 1 \\
1 & \text { side } i \text { is type } 2 \\
m L-B_{i} & \text { side } i \text { is type } 3\end{cases}
\end{aligned}
$$

Here $B i=h_{i} L / k$ is the Biot number at boundary $i$. For a derivation of the above GF for the slab-body cases $(0<$ $x<L$ ) see Reference 18 .

\section{Program TFIN}

In this section, a computer program is described that provides a formal solution for temperature in a fin by the method of GF. These formal solutions have not been stored in advance, but they are assembled, on demand, according to Equation (5).

Program TFIN is a tool for teaching and demonstrating the GF method. The program exposes students to several aspects of the GF method, including: that several types of boundary conditions that can be treated analytically; that superposition can be used to find the response to multiple simultaneous heating conditions; and, that diverse heating effects may be categorized by a numbering system. The temperature result from program TFIN is an analytical expression, and additional analysis is needed (differentiation and/or integration) in order to find numerical values for the temperature, the heat flux from the fin, and the fin efficiency.

Program TFIN, when run, displays a window that offers several choices to the student as shown in Figure 1. The student enters information through buttons or pulldown menus concerning fin geometry (infinite, semiinfinite, or finite), boundary conditions, and any internal heating. As information is entered, it is encoded in a Heat Transfer Number which is displayed at the bottom of the window. Alternately, the student may directly enter the Heat Transfer Number. Once the student has set all the desired conditions, the student must click on "Show Temperature" to direct the program to do the following: parse the displayed Heat Transfer Number for its content; assemble the requested temperature expression as a LaTeX file using an algorithm based on Equation (5); process the LaTeX file; and, display the results on screen.

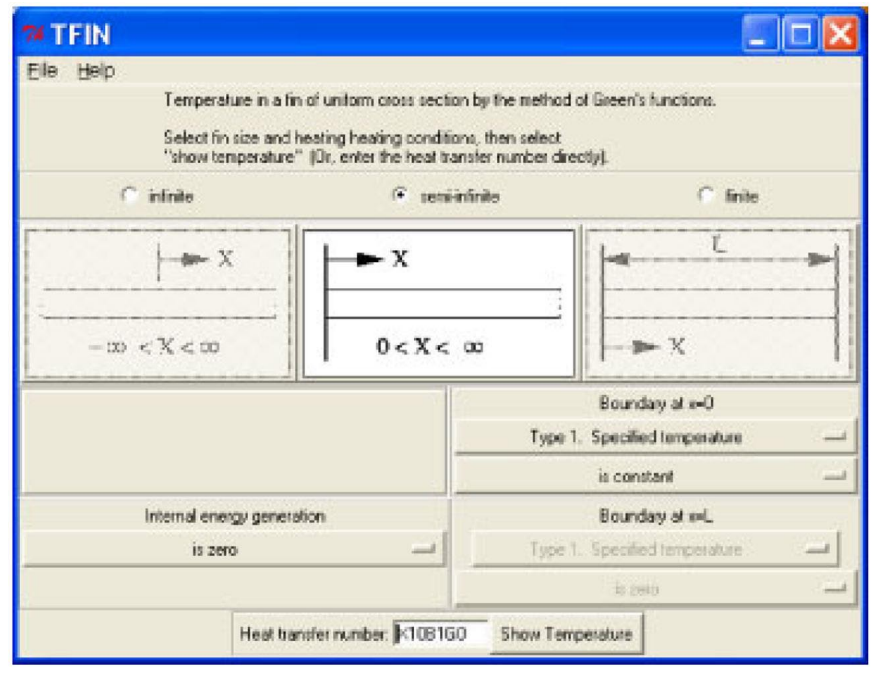

Figure 1. Main window for program TFIN provides for user input of fin size, end conditions, and internal heating condition. This information is encoded in a heat transfer number. 
Program TFIN automatically configures itself to the computer operating system, and it has been tested on computers running Linux, Win2000, and WinXP. However, program TFIN should run on any computer running Perl, Tk, and LaTeX in a sufficiently current version. All this software is generally part of the standard distributions of Linux and Unix. Windows users will have to install Perl, Tk, and LaTeX which are available at no cost if downloaded from the internet. Information on downloading and installing required software is available in the file "readme.txt" distributed with the program.

Next an example of the use of program TFIN will be given. Consider a long fin with base temperature of $T_{1}$. The sides of the fin lose heat by convection to a fluid at $T_{\infty}$. There is no internal heating. When this information is entered into program TFIN the computer display takes on the form shown in Figure 1. The GF number for this case is $\mathrm{X} 10$, and the heat transfer number is $\mathrm{X} 10 \mathrm{~B} 1 \mathrm{G} 0$. Figure 2 shows the computer display after the button "Show Temperature" is clicked. The output display includes: the differential equation for temperature; the boundary conditions; a formal expression for the temperature involving the GF designated by symbol $G_{\mathrm{X} 10}$; and, a formula for the particular GF needed for this case.

The temperature expression given by TFIN is not calculator-ready, so the student must do additional work to

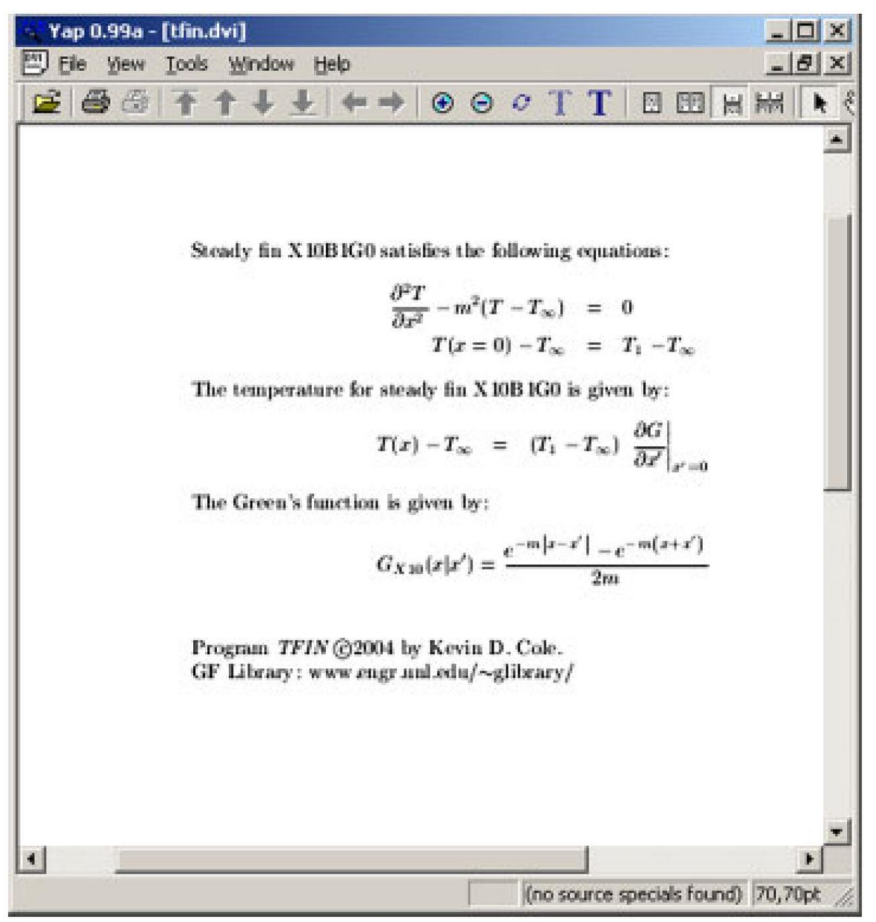

Figure 2. Output of program TFIN for the temperature in a fin, case X10B1G0, showing an expression for temperature, along with the associated differential equation, heating conditions, and Green's function. find an expression which can be evaluated numerically. For case X10B1G0, the student must differentiate the GF with respect to $x^{\prime}$ and evaluate at $x^{\prime}=0$ to find:

$$
T(x)-T_{\infty}=\left(T_{1}-T_{\infty}\right) e^{-m x}
$$

This result is given in many heat transfer texts.

This concludes the discussion of steady heat transfer in fins. In the next section, transient heat conduction in slab bodies, with no fin effect, is treated by the GF method.

\section{Heat Conduction in Slab Bodies}

In this section, unsteady heat conduction in one-dimensional planar bodies is discussed (such as walls, slabs, plane layers etc.). Side heat losses (fin losses) are not included. Consider the following boundary value problem:

$$
\begin{gathered}
\frac{\partial^{2} T}{\partial x^{2}}+\frac{1}{k} g(x, t)=\frac{1}{\alpha} \frac{\partial T}{\partial t} ; \quad x \text { in domain } R \\
\left.k_{i} \frac{\partial T}{\partial n_{i}}\right|_{x_{i}}+h_{i}\left(\left.T\right|_{x_{i}}-T_{\infty}\right)=f_{i}(t) ; \quad i=1,2 \\
\left.T\right|_{t=0}=F(x)
\end{gathered}
$$

The boundary conditions represent several different specific conditions at boundary $i$, as in the earlier discussion of fins. Additional issues, not present in the earlier discussion of fins, include: an initial condition; boundary conditions that may be a function of time; and, internal heating that may be a function of both position and time.

The temperature solution, found by the method of GF, has been discussed several times in the literature $[5,6]$, so at present the formal solution will simply be stated without further discussion. If the GF is known, the temperature solution is given by

$$
\begin{aligned}
T(x, t)= & \frac{\alpha}{k} \int_{\tau=0}^{t} \mathrm{~d} \tau \int_{x_{i}} g\left(x^{\prime}\right) G\left(x, t \mid x^{\prime}, \tau\right) \mathrm{d} x^{\prime} \\
& (\text { energy generation) } \\
& +\alpha \sum_{i=1}^{2} \int_{\tau=0}^{t} \mathrm{~d} \tau \frac{f_{i}}{k_{i}} G\left(x, t \mid x_{i}^{\prime}, \tau\right) \\
& \text { (boundary of type } 2 \text { or } 3 \text { ) } \\
& -\left.\alpha \sum_{i=1}^{2} \int_{\tau=0}^{t} \mathrm{~d} \tau f_{j} \frac{\mathrm{d} G}{\mathrm{~d} n_{j}^{\prime}}\right|_{x^{\prime}=x_{j}} \\
& \text { (boundary of type } 1 \text { only) }
\end{aligned}
$$


The GF required in Equation (12) satisfies:

$$
\begin{aligned}
\frac{\partial^{2} G}{\partial x^{2}}+\frac{1}{\alpha} \delta\left(x-x^{\prime}\right) \delta(t-\tau) & =\frac{1}{\alpha} \frac{\partial G}{\partial t} ; \quad x \text { in domain } R \\
\left.k_{i} \frac{\partial G}{\partial n_{i}}\right|_{x_{i}}+\left.h_{i} G\right|_{x_{i}} & =0 ; \quad i=1,2 \\
\left.G\right|_{t=0} & =0 .
\end{aligned}
$$

Expressions for the specific GF, cases XIJ, are given in several books (see for example References 4 and 5) and are also available on the internet site called the Greens Function Library [17].

\section{Steady Cases X00, X20, X22}

Special treatment is needed for steady heat transfer in nonfin slab bodies designated X00, X20, and X22. In these cases, the GF as defined by Equations (13)-(15) does not exist. However a modified GF may be defined by adding term $1 / V$ to the differential equation where $V$ is the volume of the domain and by relaxing the condition that the GF must vanish at infinity (see for example [3]). The modified GF may then be used in the temperature solution Equation (12), with additional constraints, as follows: the input heat to the body must satisfy an energy balance; and, the resulting temperature is unique only up to an additive constant. Physically, these constraints arise because in these geometries there is no global "heat sink" to which introduced heat can flow (there are no type 1 or 3 boundaries present). An additive constant is allowed because at physical boundaries only the derivative of temperature is specified, not the temperature itself. These constraints do not apply to fins for which the side-heat losses provide a heat sink.

\section{Program TSLAB}

In this section, a computer program is described for transient heat conduction in bodies of uniform cross section. Steady heat transfer is also included along with the special cases discussed above. Program TSLAB is similar in operation to that of program TFIN, with additional choices appropriate for the possibility of transient heat transfer, including: initial condition; time-varying boundary conditions; and, time-varying energy generation. Next an example for program TSLAB is given.

Consider a slab body, initially at uniform temperature $T_{0}$ suddenly heated at $x=0$ by heat flux $q_{1}$ and cooled at $x=L$ by convection to a fluid at temperature $T_{\infty}$. The GF number for this case is $\mathrm{X} 23$, and the heat transfer number

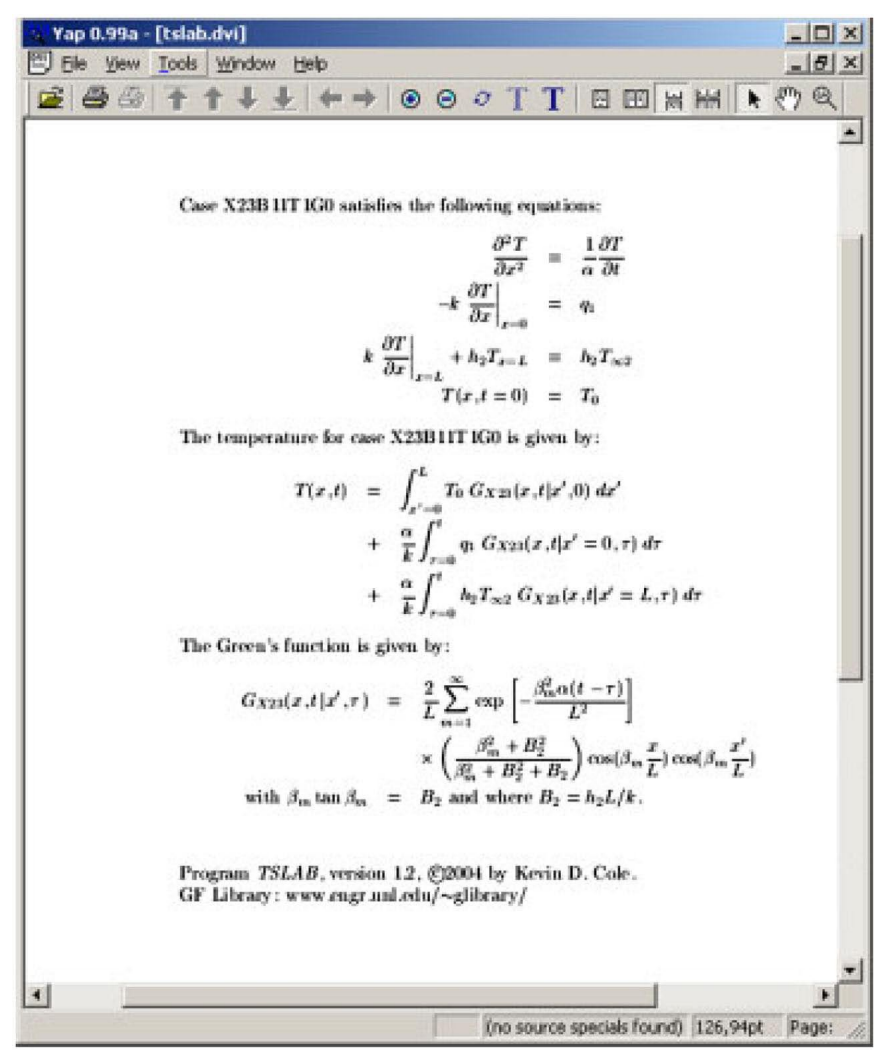

Figure 3. Output from program TSLAB for temperature as it appears on a Windows computer for case X23B11T1G0.

is X23B11T1. To save space, the input display for program TSLAB is not shown, however it is quite similar to that for TFIN with the additional choice of steady or transient heat transfer. The output display of program TSLAB for heat transfer number X23B11T1 is shown in Figure 3 . The boundary value problem for temperature is given, followed by an integral expression for the temperature and a series expression for $G_{\mathrm{X} 23}$.

\section{Open-Source Software}

Programs TFIN and TSLAB produce output in LaTeX format. LaTeX is the typesetting standard for the American Mathematical Society, and it is available for free for a variety of computer operating systems. LaTeX was also used because the GF were already available in this format in the GF Library [17]. Programs TFIN and TSLAB were written in Perl with graphics library Tk. Perl was chosen both for its strength with string manipulation and because it is an open-source language.

An open-source language was specifically chosen for this project because it will run on a variety of computer operating systems and supporting software needed by the user is available at low (or zero) cost. Cost is partic- 
ularly important to students and educators. Open-source software was also chosen, in part, to avoid putting too much "stock" into a single software company. The author intends to develop additional programs in the coming years, perhaps in the coming decades, and to invest time and effort in software that depends upon the financial health of a single software company is risky. Just as a financial portfolio should be diversified to reduce risk, open-source software is supported by a diverse community of users and software developers that are independent of any single software company.

Open-source code is not for everyone, however. Programs written in an interpreted language such as Perl/ Tk generally must be distributed as source code, not as a compiled binary. Since anyone can look at the source code, the intellectual property is "given away" with the program, and generally such code does not produce royalties.

Programs TFIN and TSLAB are distributed as free software. The programs are copyrighted by the author and distributed under the GNU General Public License, with the intention that the programs will remain available for re-distribution and/or modification by anyone in the hope that it will be useful, but without any warranty whatsoever [19].

\section{Math Standards for Computers}

Several educators, upon seeing programs TFIN and TSLAB, have asked if a version exists in their favorite symbolic software such as Maple, Mathematica, etc. The answer is no, as the author has chosen open-source software as discussed above. However, anyone is free to alter programs TFIN and TSLAB, under the General Public License, to add a "translation module" to port the output into a symbolic program. This would produce a narrow application tailored to a single symbolic program. A broader solution would be possible if there were a standard syntax for math that all symbolic math programs could use. Although typesetting standard LaTeX is useful for display of math, what is needed is a standard syntax for math content. As the world wide web has become a potent force for cross-platform software development, the future of math and computers probably lies with web browsers.

In February 2001 version 2.0 of MathML, which stands for mathematics markup language, was announced as a recommendation by the World Wide Web Consortium [20]. MathML is likely to evolve into a widely accepted standard for display of math and for encoding math content on the world wide web [21]. The latest version of several web browsers already support some aspects of MathML, but more development is needed. For example, MathML is too verbose for people to write directly (except for the simplest expressions), so that authoring tools will also be needed before MathML can be widely used. Several authoring tools are under development or are in beta testing [22].

The author's hope is that MathML translation software will soon be developed to convert LaTeX into MathML, and that many symbolic manipulation programs will soon accept MathML as an option for importing data. How soon will this be possible? Early versions of LaTeX to MathML translation tools for math display are already under development by the open-source community. A few proprietary conversion tools already exist as browser plug-ins that produce MathML output. Several companies that sell symbolic manipulation programs are publicly committed to support of MathML in their products, and some math programs currently support MathML output for display in a web browser. However, the "import" of MathML into symbolic math programs may have to wait for additional development of the MathML standard for math content.

\section{Conclusion}

Exact solutions will always play a role in engineering practice for checking purposes and for precisely quantifying the accuracy of fully numeric codes. Students need to develop a balanced understanding of the uses of both analytic and numeric methods. In this paper, the GF method is discussed for fins and one-dimensional slab bodies, material usually taught in an undergraduate heat transfer course. Computer programs TFIN and TSLAB are intended to expose students to the breadth of conditions that may be treated analytically in the hope that they will be better informed on the use of both analytic and numerical methods.

\section{Acknowledgments}

The author thanks James Beck for encouragement of this work and acknowledges funding from the University of Nebraska Foundation.

\section{Appendix: GF Solution for Fins}

In this appendix, the temperature solution in fins is derived with the GF method. The derivation begins with 
the reciprocity relation applied to the differential equation for the GF:

$$
\frac{\mathrm{d}^{2} G}{\mathrm{~d} x^{\prime 2}}-m^{2} G-\delta\left(x^{\prime}-x\right)=0
$$

The next step is to rewrite the temperature equation with a simple change of variables $x \rightarrow x^{\prime}$ and let $\theta=T\left(x^{\prime}\right)$ $-T_{\infty}$ to give:

$$
\frac{\mathrm{d}^{2} \theta}{\mathrm{d} x^{\prime 2}}-m^{2} \theta+\frac{1}{k} g\left(x^{\prime}\right)=0
$$

Multiply Equation (16) by $\theta$ and multiply Equation (17) by $G$ and subtract, the result is:

$$
\begin{aligned}
G \frac{\mathrm{d}^{2} \theta}{\mathrm{d} x^{\prime 2}} & -\theta \frac{\mathrm{d}^{2} G}{\mathrm{~d} x^{\prime 2}}-G m^{2} \theta+\theta m^{2} G \\
& +\frac{1}{k} g\left(x^{\prime}\right) G-\theta \delta\left(x^{\prime}-x\right)=0 ;
\end{aligned}
$$

Note that the term involving $m^{2}$ cancels. Now integrate with respect to $x^{\prime}$ over domain $R$ and use Table 1, property 3 of the Dirac delta function (sometimes called the sifting property) to find:

$$
\begin{aligned}
T(x)-T_{\infty}= & \int_{R}\left[G \frac{\mathrm{d}^{2} \theta}{\mathrm{d} x^{\prime 2}}-\theta \frac{\mathrm{d}^{2} G}{\mathrm{~d} x^{\prime 2}}\right] \mathrm{d} x^{\prime} \\
& +\frac{1}{k} \int_{R} g\left(x^{\prime}\right) G\left(x^{\prime} \mid x\right) \mathrm{d} x^{\prime}
\end{aligned}
$$

The second term is the contribution to the temperature from the energy generation in the form of a superposition integral over the domain. The first integral term in the above equation will now be simplified. Consider just the first integral term from Equation (19) and perform integration by parts (in two- or three-dimensional bodies Green's theorem is needed):

$$
\begin{aligned}
& \int_{x^{\prime}=x_{1}}^{x_{2}}\left[G \frac{\mathrm{d}^{2} \theta}{\mathrm{d} x^{\prime 2}}-\theta \frac{\mathrm{d}^{2} G}{\mathrm{~d} x^{\prime 2}}\right] \mathrm{d} x^{\prime} \\
& =\left[G \frac{\mathrm{d} \theta}{\mathrm{d} x^{\prime}}-\theta \frac{\mathrm{d} G}{\mathrm{~d} x^{\prime}}\right]_{x^{\prime}=x_{1}}^{x_{2}}-\int_{x^{\prime}=x_{1}}^{x_{2}}\left[\frac{\mathrm{d} \theta}{\mathrm{d} x^{\prime}} \frac{\mathrm{d} G}{\mathrm{~d} x^{\prime}}-\frac{\mathrm{d} G}{\mathrm{~d} x^{\prime}} \frac{\mathrm{d} \theta}{\mathrm{d} x^{\prime}}\right] \mathrm{d} x^{\prime}
\end{aligned}
$$

For the moment, domain $R$ has been replaced by domain $\left(x_{1}, x_{2}\right)$ so that two boundaries can be discussed. Later, notation to include semi-infinite and infinite domains will be re-introduced. In the equation above, the term in brackets is evaluated at the boundaries and the remaining integral term is zero.

The boundary terms in the above expression can now be simplified with the boundary conditions for $G$ and $\theta$, however, the type of boundary condition influences the form of the solution. For boundary conditions of type
2 or 3 , the boundary conditions given by Equations (2) and (4) may be written:

$$
\begin{gathered}
\left.\frac{\mathrm{d} \theta}{\mathrm{d} n_{i}}\right|_{x_{i}}=-\frac{h_{i}}{k_{i}} \theta\left(x_{i}\right)+\frac{f_{i}}{k_{i}} ; \quad i=1,2 \\
\left.\frac{\mathrm{d} G}{\mathrm{~d} n_{i}}\right|_{x_{i}}=-\frac{h_{i}}{k_{i}} G ; \quad i=1,2
\end{gathered}
$$

Now the notation for the outward-normal direction in the boundary conditions finds its use. Replace the above boundary conditions into Equation (20), and remember that there is a sign change at $x^{\prime}=x_{1}$ where $\mathrm{d} / \mathrm{d} n^{\prime}{ }_{1}=$ $-\mathrm{d} / \mathrm{d} x^{\prime}$ :

$$
\begin{aligned}
{\left[G \frac{\mathrm{d} \theta}{\mathrm{d} x^{\prime}}-\theta \frac{\mathrm{d} G}{\mathrm{~d} x^{\prime}}\right]_{x^{\prime}=x_{1}}^{x_{2}}=} & G\left[-\frac{h_{2}}{k_{2}} \theta+\frac{f_{2}}{k_{2}}\right]-\theta\left[-\frac{h_{2}}{k_{2}} G\right] \\
& -\left\{G\left[\frac{h_{1}}{k_{1}} \theta-\frac{f_{1}}{k_{1}}\right]-\theta\left[\frac{h_{1}}{k_{1}} G\right]\right\} \\
= & \sum_{i=1}^{2} \frac{h_{i}}{k_{i}} G
\end{aligned}
$$

Note that all terms containing $\theta$ have canceled, and that the two remaining boundary terms have been combined together into a single sum representing the effect on temperature of boundaries of type 2 or 3 .

For boundary conditions of type 1 the boundaries must be treated differently. Go back to Equation (20) and use the fact that on type 1 boundaries $G=0$ and $\theta=$ $f_{j}, j=1,2$, so that

$$
\left[G \frac{\mathrm{d} \theta}{\mathrm{d} x^{\prime}}-\theta \frac{\mathrm{d} G}{\mathrm{~d} x^{\prime}}\right]_{x^{\prime}=x_{1}}^{x_{2}}=-\sum_{j=1}^{2} f_{i} \frac{\partial G}{\partial n_{j}^{\prime}}
$$

Here the outward normal, $n_{j}$, is used to determine the sign of the boundary terms.

Now the temperature expression can be assembled into a general form by combining Equations (19), (23), and (24) to give:

$$
\begin{aligned}
T(x)-T_{\infty}= & \frac{1}{k} \int g\left(x^{\prime}\right) G\left(x \mid x^{\prime}\right) \mathrm{d} x^{\prime} \\
& \text { (for energy generation) } \\
& +\sum_{i=1}^{2} \frac{f_{i}}{k_{i}} G\left(x \mid x_{i}^{\prime}\right)
\end{aligned}
$$

(for boundary of type 2 or 3 )

$$
-\left.\sum_{i=1}^{2} f_{j} \frac{\mathrm{d} G}{\mathrm{~d} n_{j}^{\prime}}\right|_{x^{\prime}=x_{j}}
$$

(for boundary of type 1 only)

This is the GF solution for fins stated earlier in Equation (5). 


\section{References}

[1] H. S. Carslaw, Mathematical theory of the conduction of heat in solids, Macmillan, New York, 1921.

[2] H. S. Carslaw and J. C. Jaeger, Conduction of heat in solids, Oxford University Press, Oxford, UK, 1959.

[3] G. Barton, Elements of Green's Functions and Propagation, Oxford University Press, Oxford, UK, 1989.

[4] Y. A. Melnikov, Green's functions in applied mechanics, Computational Mechanics Publications, Boston- Southhampton, 1995.

[5] J. V. Beck, K. D. Cole, A. Haji-Sheikh, and B. Litkouhi, Heat conduction using Green's functions, Hemisphere Publishing Corporation, Washington, DC, 1992.

[6] D. G. Duffy, Green's functions with applications, Chapman and Hall/CRC, Boca Raton, FL, 2001.

[7] D. R. Harper and P. W. Brown, Mathematical equations for heat conduction in the fins of air-cooled engines, NACA Report 158 (1922).

[8] K. A. Gardner, Efficiency of extended surfaces, Trans ASME 67 (1945), 621-631.

[9] M. Jakob, Heat transfer, Wiley, New York, 1949.

[10] A. Haji-Sheikh, L. Yan, and S. P. Kinsey, A single equation solution for conduction in fins, Int J Heat Mass Transfer 34 (1991), 159-165.

[11] Intellipro, Interactive heat transfer, Wiley, New York, 1996.

[12] A. Haji-Sheikh, Conduction Solver, Lab Report, Department of Mechanical Engineering, University of Texas at Arlington, 1989.
[13] A. Haji-Sheikh, Heat diffusion in heterogeneous media using heat-flux-conserving basis functions, J Heat Transfer 110 (1988), 276-282.

[14] R. J. Ribando and G. W. O'Leary, Teaching module for onedimensional transient conduction, Comput Appl Eng Educ 6 (1998), 41-51.

[15] C. W. Somerton, L. J. Genik, D. Beavers, and T. Jammer, A Nusselt number correlation classification system, Proceedings of the American Society of Engineering Education, 1999.

[16] K. D. Cole and P. E. Crittenden, Heat Conduction in Cartesian Coordinates and a Library of Green's Functions, 35th National Heat Transfer Conference, Anaheim, CA, June 10-12, 2001.

[17] K. D. Cole, Green's Function Library, http://www.engr.unl. edu/glibrary

[18] P. E. Crittenden and K. D. Cole, Fast-converging steady-state heat conduction in the rectangular parallelepiped, Int $J$ Heat Mass Transfer 45 (2002), 3585-3596.

[19] GNU Free Software Foundation, http://www.gnu.org/fsf/fsf. html (accessed Sept. 3, 2003).

[20] http://www.w3.org/Math/ (accessed June 17, 2003).

[21] R. Miner and P. Topping, Math on the web: A status report, Proceedings of the Second International MathML Conference, September, 2002, http://www.mathtype. com/en/reference/webmath/status/status_Sep_02.htm

[22] http://www.w3.org/Math/implementations.html (accessed June 17, 2003).

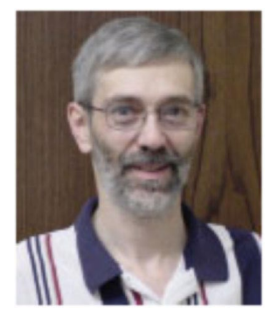

Biography

Kevin Cole is an associate professor of mechanical engineering at the University of Nebraska-Lincoln. Dr. Cole's research interests include Green's functions, heat transfer, fluid-flow measurement, and inverse problems. He studied at Iowa State (BS 1977), University of Minnesota (MS 1979), and Michigan State University (PhD 1986). Before moving to Nebraska he worked for Deere and Company (Waterloo, IA) and taught at Trinity College (Hartford, CT). Dr. Cole is a member of ASEE, ASME, AIAA, and Sigma $\mathrm{Xi}$, and he is a registered professional engineer. He can be reached at kcole1@unl.edu 